\title{
Preparation of 3,4-dihydro-2,1-benzothiazine 2,2-dioxides with polymer-supported hypervalent iodine reagents
}

\author{
Kenji Sakuratani $^{a}$ and Hideo Togo $*^{a, b}$ \\ ${ }^{a}$ Graduate School of Science and Technology, Chiba University, \\ ${ }^{b}$ Department of Chemistry, Faculty of Science, Chiba University, \\ Yayoi-cho 1-33, Inage-ku, Chiba 263-8522, Japan \\ E-mail: togo@scichem.s.chiba-u.ac.jp
}

\section{Dedicated to Professor Anastasios Varvoglis on the occasion of his $65^{\text {th }}$ birthday \\ (received 28 Jan 03; accepted 24 Feb 03; published on the web 04 Mar 03)}

\begin{abstract}
Radical cyclization and ionic cyclization onto the aromatic rings of 2-arylethanesulfonamides with polymer-supported hypervalent iodine reagents were examined, where the reactivities appear to be dependent on the substituent bonded to the nitrogen atom of 2arylethanesulfonamides to obtain the corresponding 3,4-dihydro-2,1-benzothiazine 2,2-dioxides.
\end{abstract}

Keywords: 3,4-Dihydro-2,1-benzothiazine 2,2-dioxide, cyclization, polymer-supported hypervalent iodine reagent, recycle

\section{Introduction}

Extensive study on organohypervalent iodine compounds has been carried out and these compounds have been widely used for organic synthesis. ${ }^{1}$ Based on the synthetic utility of the organohypervalent iodine reagents, some polymer-supported hypervalent iodine reagents have been also developed and their synthetic uses were studied. ${ }^{2-4}$ As one important synthetic application with organohypervalent iodine reagents, we have been studying the preparation of heterocyclic compounds via radical reaction mechanism with (diacetoxyiodo)arene in the presence of iodine. ${ }^{5}$ Among them, 3,4-dihydro-2,1-benzothiazine 2,2-dioxide derivatives have potent biological activities such as lipoxygenase inhibition and as drugs for heart diseases (Fig. 1). Therefore, active studies for the synthetic development of benzothiazine derivatives have been carried out. ${ }^{6}$ 


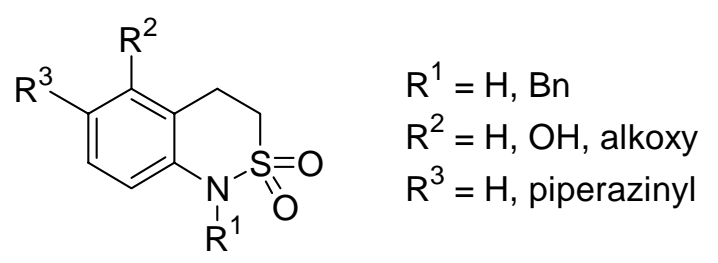

Figure 1. 3,4-Dihydro-2,1-benzothiazine 2,2-Dioxide Derivatives.

Today, five established methods for the construction of the 3,4-dihydro-2,1-benzothiazine 2,2dioxide skeleton are known, i.e.:

i) cyclization of 2-(o-aminophenyl)ethanesulfonic acid with phosphoryl chloride ${ }^{7 \mathrm{a}}$

ii) cyclization of $N$-phenylsulfamoylacetic acid with polyphosphoric acid and subsequent reduction of the carbonyl group ${ }^{7 b}$

iii) pyrolysis of $\beta$-arylethanesulfonyl azides ${ }^{7 \mathrm{c}}$

iv) cyclization of $N$-benzyl- $N$-methanesulfonyl(o-chloromethyl)aniline with sodium hydride $^{7 \mathrm{~d}}$

v) radical cyclization of 2-arylethanesulfonamides with (diacetoxyiodo)benzene (DIB) and iodine $^{5 \mathrm{e}}$

However, methods $\mathrm{i} \sim$ iv require many preparative steps, and quite acidic or basic conditions; moreover, the yields of cyclization products are generally not so high. On the other hand, method $\mathrm{v}$ provides the corresponding cyclization products in good yields under mild conditions. Based on this method $\mathrm{v}$, we planned to develop the preparation of 3,4-dihydro-2,1-benzothiazine 2,2dioxides from 2-arylethanesulfonamides with polymer-supported hypervalent iodine reagents.

\section{Results and Discussion}

\section{Cyclization of $\boldsymbol{N}$-methyl-2-arylethanesulfonamides via radical pathway}

Based on the direct preparation of $N$-methyl-3,4-dihydro-2,1-benzothiazine 2,2-dioxides from $N$ methyl-2-arylethanesulfonamides with (diacetoxyiodo)benzene (DIB) in the presence of iodine under photochemical conditions ${ }^{5 \mathrm{e}}$, the same radical cyclization with poly\{4(diacetoxyiodo)styrene (PSDIB) in the presence of iodine was carried out. Thus, irradiation of a mixture of $N$-methyl-2-arylethanesulfonamides with $\operatorname{PSDIB}^{3 \mathrm{a}}$ (3.0 eq.) in the presence of iodine (1.0 eq.) in 1,2-dichloroethane with a tungsten lamp $(500 \mathrm{~W})$ was carried out at room temperature, and the results are shown in Table 1 and Scheme 1.

Table 1. Radical cyclization of $N$-methyl-2-arylethanesulfonamides 


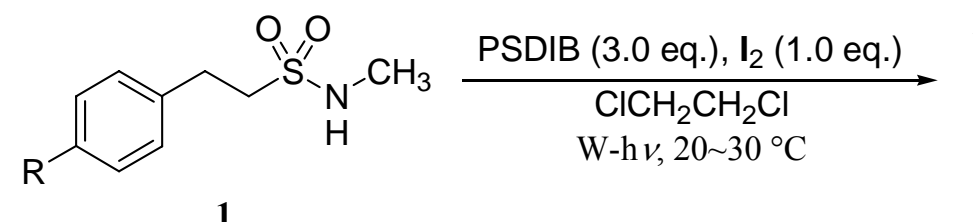

\begin{tabular}{ccccc}
\hline Entry & Substrate & R & Time (h) & \multicolumn{1}{c}{ Yields (\%) } \\
\hline 1 & 1a & H & 2 & $96(25: 71)$ \\
2 & 1b & F & 3 & $88(35: 43)$ \\
3 & 1c & Cl & 3 & $61(30: 31)$ \\
4 & 1d & $\mathrm{CH}_{3}$ & 3 & $95(0: 95)$ \\
5 & 1a & H & 2 & $40(0: 40)^{\mathrm{a}, \mathrm{b}}$ \\
\hline
\end{tabular}

${ }^{\text {a }}$ PSHTIB was used instead of PSDIB.

${ }^{\mathrm{b}}$ Starting material was recovered in $27 \%$ yield.<smiles>CNS(=O)(=O)CCc1cccc2ccccc12</smiles><smiles>CNS(=O)(=O)CCc1cn(S(=O)(=O)O)c2ccccc12</smiles>

Scheme 1. Radical cyclization of $N$-methyl-2-( $\alpha$-naphthyl)ethanesulfonamide and $N$-methyl-2$\{3$ '-[N-(benzenesulfonyl)indolyl $]\}$ ethanesulfonamide.

In spite of the heterogeneous solution, the photolytic radical reaction proceeds effectively to provide the corresponding cyclization products in good yields via sulfonamidyl radicals, with 2phenyl-, 2-naphthyl-, and 2-indolylethanesulfonamides. When poly\{4[hydroxy(tosyloxy)iodo]styrene $\}$ (PSHTIB) ${ }^{4 \mathrm{f}}$ was used instead of PSDIB under the same conditions, the yield of cyclization product was decreased (entry 5). The same photolytic treatment of $\mathrm{N}$-methoxy-2-arylethanesulfonamides with PSDIB in the presence of iodine did not proceed at all. 


\section{Cyclization of $N$-methoxy-2-arylethanesulfonamides via ionic pathway}

Then, ionic cyclization of 2-arylethanesulfonamides with PSDIB and PSHTIB was carried out as shown in Table 2. The cyclization of $N$-methyl-2-phenylethanesulfonamides with PSDIB (entry 1) and PSHTIB did not proceed at all, and the starting materials were recovered. However, treatment of $\mathrm{N}$-methoxy-2-arylethanesulfonamides with PSHTIB in acetonitrile gave the corresponding cyclization products, $N$-methoxy-3,4-dihydro-2,1-benzothiazine 2,2-dioxides in good to moderate yields at room temperature (entries 2-5). As shown in Table 2, electrondonating groups on the aromatic ring increase the yield, and electron-withdrawing groups on the aromatic ring reduce the yield of the cyclization products. This suggests that the reaction proceeds through an electrophilic pathway onto the aromatic ring of $\mathrm{N}$-methoxy-2arylethanesulfonamides.

Table 2. Ionic cyclization of $N$-methoxy-2-arylethanesulfonamides

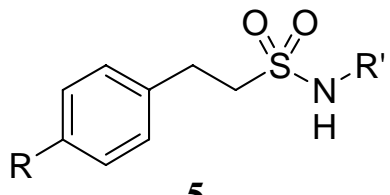

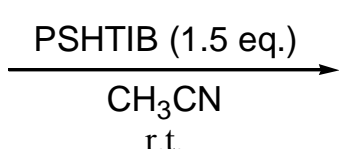

r.t.<smiles>[R]c1ccc2c(c1)N([R])S(=O)(=O)CC2</smiles>

6

\begin{tabular}{cccccc}
\hline Entry & Substrate & $\mathrm{R}$ & $\mathrm{R}^{\prime}$ & Time (h) & Yields (\%) \\
\hline 1 & $\mathbf{1 a}$ & $\mathrm{H}$ & $\mathrm{CH}_{3}$ & 6 & $0^{\mathrm{a}}$ \\
2 & $\mathbf{5 a}$ & $\mathrm{H}$ & $\mathrm{OCH}_{3}$ & 4 & $64[70]^{\mathrm{b}}$ \\
3 & $\mathbf{5 b}$ & $\mathrm{F}$ & $\mathrm{OCH}_{3}$ & 4 & 31 \\
4 & $\mathbf{5 c}$ & $\mathrm{Cl}$ & $\mathrm{OCH}_{3}$ & 4 & 34 \\
5 & $\mathbf{5 d}$ & $\mathrm{CH}_{3}$ & $\mathrm{OCH}_{3}$ & 4 & 67 \\
6 & $\mathbf{5 a}$ & $\mathrm{H}$ & $\mathrm{OCH}_{3}$ & 6 & trace $^{\mathrm{c}}$ \\
\hline
\end{tabular}

${ }^{a}$ Starting material was quantitatively recovered.

${ }^{\mathrm{b}}$ Regenerated PSHTIB was used.

${ }^{\mathrm{c}}$ PSDIB was used instead of PSHTIB.

The same treatment of $\mathrm{N}$-methoxy-2-phenylethanesulfonamides with PSDIB, which has a less polarized iodine center than that of PSHTIB, did not generate any cyclization product (entry 6). When $N$-methoxy-2-(4'-methoxyphenyl)ethanesulfonamide, which has an electron-rich aromatic ring, was used, this reaction provides the corresponding spiro sultam (Scheme 2). 


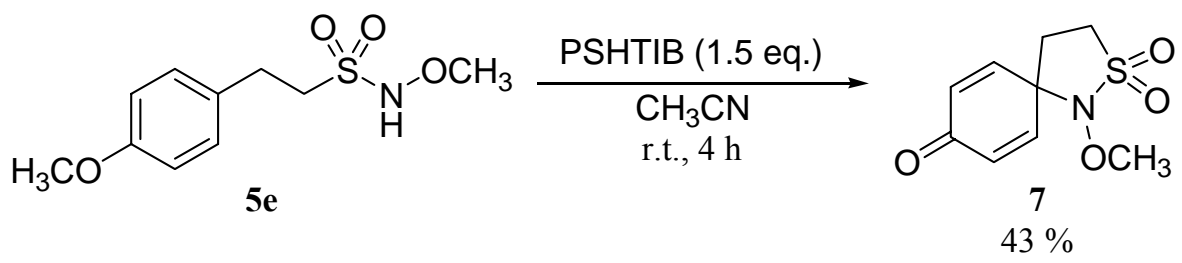

Scheme 2. Ionic cyclization of $N$-methoxy-2-(4'-methoxyphenyl)ethanesulfonamide.

Thus, under the ionic conditions, the $N$-methoxy group in $N$-methoxy-2-arylethanesulfonamides plays an important role for the formation of an N-I bonded hypervalent iodine intermediate, and the subsequent electrophilic cyclization onto the aromatic ring by the electrophilic nitrogen atom proceeds smoothly to generate $N$-methoxy-3,4-dihydro-2,1-benzothiazine 2,2-dioxide, together with the formation of poly(4-iodostyrene) and $p$-toluenesulfonic acid. The recovered poly(4iodostyrene) can be converted to PSDIB and PSHTIB, and reused for the same reactions (Table 2, entry 2).

$\mathrm{N}$-Methoxy-3-arylpropionamides react with [bis(trifluoroacetoxy)iodo]benzene to form $\mathrm{N}-\mathrm{I}$ bonded hypervalent iodine intermediates in which the electron-deficient nitrogen atom is attacked by the $O$-position of the side-chained aromatic ring to give $N$-methoxy-3,4-dihydro- $1 H$ quinolin-2-ones. ${ }^{8}$ Based on the results, treatment of $N$-methoxy-3-phenylpropaneamide with PSHTIB was carried out to provide $N$-methoxy-3,4-dihydro- $1 H$-quinolin-2-one in moderate yield.<smiles>CONC(=O)CCc1ccccc1</smiles>

8

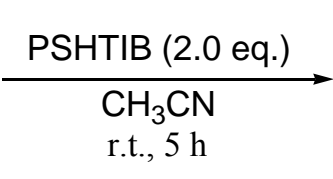

r.t., $5 \mathrm{~h}$

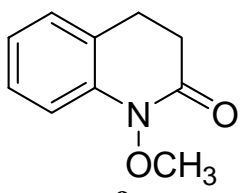

9

Scheme 3. Ionic cyclization of $N$-methoxy-3-phenylpropionamide.

Once $\mathrm{N}$-methoxy-3,4-dihydro- $1 \mathrm{H}$-quinolin-2-one and $\mathrm{N}$-methoxy-3,4-dihydro-2,1-benzothiazine 2,2-dioxides are formed, the corresponding $N$-free 3,4-dihydro- $1 H$-quinolin-2-one and $N$-free 3,4-dihydro-2,1-benzothiazine 2,2-dioxides are easily obtained in quite good yields with samarium iodide in tetrahydrofuran.

\section{Experimental Section}

General Procedures. Melting points were determined on an electrothermal apparatus in open capillary tubes and are uncorrected. ${ }^{1} \mathrm{H}$ NMR and ${ }^{13} \mathrm{C}$ NMR spectra were obtained on a JEOLJMN-GSX 500 and a JEOL-JMN-GSX 400. Chemical shifts are expressed in parts per million from tetramethylsilane (TMS) in $\delta$ units. $J$ values are given in hertz. IR spectra were recorded on 
a FT/IR-200 spectrometer. Mass spectra were measured with QMS (EI) and high-resolution mass spectra (HRMS) were measured with a 110A mass spectrometer. Microanalyses of polymers were performed with Perkin-Elmer 240B and 240 elemental analyzers at the Chemical Analysis Center of Tsukuba University. Silica Gel 60 (Kanto Kagaku Co.) was used for column chromatography, Kieselgel 60 F254 (Merck) was used for TLC, and Wakogel B-5F was used for preparative TLC. All reactions were carried out under an argon atmosphere.

Materials. Most sulfonamides and amide were prepared by the reaction of sulfonyl chlorides or acid chloride with amines. Sulfonyl chlorides were prepared from the corresponding sodium sulfonates, which were obtained from the reaction of alkyl bromides and sodium sulfite. ${ }^{9}$

Preparation of poly[4-(diacetoxyiodo)styrene] (PSDIB). A mixture of $16 \mathrm{~g}$ (153 mmol) of polystyrene (Aldrich 33165-1), $18 \mathrm{~g}$ (71 mmol) of iodine, $7 \mathrm{~g}$ (21 mmol) of iodine pentoxide, 40 $\mathrm{mL}$ of carbon tetrachloride, and $35 \mathrm{~mL}$ of $50 \%$ sulfuric acid in $200 \mathrm{~mL}$ of nitrobenzene was kept at $90{ }^{\circ} \mathrm{C}$ for $72 \mathrm{~h}$. After the reactions were completed, the reaction mixture was diluted with 100 $\mathrm{mL}$ of chloroform and precipitation occurred upon the addition of methanol $(1500 \mathrm{~mL})$. The precipitates were collected by filtration to give poly(4-iodostyrene). IR peaks of 700 and 760 $\mathrm{cm}^{-1}$ in polystyrene disappeared, and a new peak at $820 \mathrm{~cm}^{-1}$ appeared in poly(4-iodostyrene). Hydrogen peroxide $(30 \%, 40 \mathrm{~mL})$ was added dropwise to an acetic anhydride solution $(145 \mathrm{~mL})$ at $0{ }^{\circ} \mathrm{C}$. The solution was slowly warmed to room temperature and stirred overnight. To this solution was added poly(4-iodostyrene) ( $8 \mathrm{~g}$ ). The solution was kept at about $48{ }^{\circ} \mathrm{C}$ overnight. At the end of the reaction, diethyl ether was added to the solution to cause precipitation. The product (ca. 9 g) was collected by filtration: IR (KBr) 1630 (br), 1560 (br), 1480, 1450, 1410, 1260, 1180, 1000, 770 (br) $\mathrm{cm}^{-1}$; Anal. Found: C, 36.33; H, 3.66; I, 39.19.

Preparation of poly\{[4-hydroxy(tosyloxy)iodo]styrene\} (PSHTIB). PSDIB (10 mmol) was added to a solution of $p$-toluenesulfonic acid monohydrate $(20 \mathrm{mmol})$ in chloroform $(20 \mathrm{~mL})$ and stirred for $24 \mathrm{~h}$ at room temperature under dark conditions. After the reaction was completed, diethyl ether $(50 \mathrm{~mL})$ was added to the reaction mixture. The mixture was filtered, and the solid was washed with diethyl ether: IR (KBr) 3430 (br), 2920, 1480, 1400, 1210, 1180, 1130, 1040, 1010, 820, 765, 700, $570 \mathrm{~cm}^{-1}$; Anal. Found: C, 36.33; H, 3.66.

\section{Typical procedure for the radical cyclization of $N$-methyl-2-phenylethanesulfonamide with PSDIB}

PSDIB (1.5 mmol) was added to a solution of $N$-methyl-2-phenylethanesulfonamide $(0.5 \mathrm{mmol})$ in 1,2-dichloroethane $(5 \mathrm{~mL})$, and then iodine $(0.5 \mathrm{mmol})$ was added to the solution. The mixture was irradiated with a tungsten lamp $(500 \mathrm{~W})$ at $20-30{ }^{\circ} \mathrm{C}$ for $2 \mathrm{~h}$ under an argon atmosphere. After the reaction, the reaction mixture was filtered. The filtrate was poured into a saturated aqueous sodium sulfite solution and extracted with chloroform thrice. The organic layer was dried over sodium sulfate. After removal of the solvent under reduced pressure, the residue was chromatographed on silica gel (eluent: hexane / ethyl acetate $=3 / 1$ ). 
$\boldsymbol{N}$-Methyl-3,4-dihydro-2,1-benzothiazine 2,2-dioxide (2a). mp 77-79 ${ }^{\circ} \mathrm{C}$; IR ( $\mathrm{KBr}$ ) 2980, 2940, 1580, 1490, 1330, $1170 \mathrm{~cm}^{-1} ;{ }^{1} \mathrm{H} \mathrm{NMR}\left(\mathrm{CDCl}_{3}\right) \delta 3.30(\mathrm{~s}, 3 \mathrm{H}), 3.33(\mathrm{t}, J=6.9 \mathrm{~Hz}, 2 \mathrm{H})$, $3.44(\mathrm{t}, J=6.9 \mathrm{~Hz}, 2 \mathrm{H}), 6.96(\mathrm{dd}, J=8.0,1.0 \mathrm{~Hz}, 1 \mathrm{H}), 7.05(\mathrm{td}, J=7.6,1.0 \mathrm{~Hz}, 1 \mathrm{H}), 7.16(\mathrm{dd}, J$ = 7.6, 1.2 Hz, 1H), $7.27(\mathrm{~m}, 1 \mathrm{H}) ;{ }^{13} \mathrm{C} \mathrm{NMR}\left(\mathrm{CDCl}_{3}\right) \delta 27.90(\mathrm{~s}), 31.97(\mathrm{p}), 45.55(\mathrm{~s}), 117.47(\mathrm{t})$, $122.82(\mathrm{q}), 123.14(\mathrm{t}), 127.88(\mathrm{t}), 129.30$ (t), $141.14(\mathrm{q})$; MS (EI) $\mathrm{M}^{+}$197; Anal. Calcd for $\mathrm{C}_{9} \mathrm{H}_{11} \mathrm{NO}_{2} \mathrm{~S}: \mathrm{C}, 54.80 ; \mathrm{H}, 5.62 ; \mathrm{N}, 7.10$. Found: $\mathrm{C}, 54.74 ; \mathrm{H}, 5.52 ; \mathrm{N}, 7.07$.

$\boldsymbol{N}$-Methyl-6-iodo-3,4-dihydro-2,1-benzothiazine 2,2-dioxide (3a). mp $139-141{ }^{\circ} \mathrm{C}$; IR ( $\mathrm{KBr}$ ) 2940, 1590, 1560, 1320, $1100 \mathrm{~cm}^{-1}$; ${ }^{1} \mathrm{H}$ NMR $\left(\mathrm{CDCl}_{3}\right) \delta 3.26(\mathrm{~s}, 3 \mathrm{H}), 3.32(\mathrm{t}, J=7.1 \mathrm{~Hz}, 2 \mathrm{H})$, 3.40 (t, $J=7.1 \mathrm{~Hz}, 2 \mathrm{H}), 6.70(\mathrm{~d}, J=8.8 \mathrm{~Hz}, 1 \mathrm{H}), 7.49$ (d, $J=2.0 \mathrm{~Hz}, 1 \mathrm{H}), 7.55$ (dd, $J=8.8,2.0$ $\mathrm{Hz}, 1 \mathrm{H}) ;{ }^{13} \mathrm{C} \mathrm{NMR}\left(\mathrm{CDCl}_{3}\right) \delta 27.51(\mathrm{~s}), 31.74(\mathrm{p}), 45.27(\mathrm{~s}), 86.24(\mathrm{q}), 119.16(\mathrm{t}), 125.03(\mathrm{q})$, 136.73 (t), 137.89 (t), 141.06 (q); MS (EI) $\mathrm{M}^{+}$323; Anal. Calcd for $\mathrm{C}_{9} \mathrm{H}_{10} \mathrm{INO}_{2} \mathrm{~S}$ : C, 33.45; H, 3.12 ; N, 4.33. Found: C, 33.55; H, 3.06; N, 4.27 .

$\mathrm{N}$-Methyl-7-fluoro-3,4-dihydro-2,1-benzothiazine 2,2-dioxide (2b). mp $67-68{ }^{\circ} \mathrm{C}$; IR ( $\mathrm{KBr}$ ) $3000,2950,1620,1590,1510,1320,1300,1210,1170,1140 \mathrm{~cm}^{-1} ;{ }^{1} \mathrm{H} \mathrm{NMR}\left(\mathrm{CDCl}_{3}\right) \delta 3.28(\mathrm{~s}$, $3 \mathrm{H}), 3.34$ (ddd, $J=7.5,6.3,1.3 \mathrm{~Hz}, 2 \mathrm{H}), 3.43$ (ddd, $J=7.5,6.3,1.3 \mathrm{~Hz}, 2 \mathrm{H}), 6.66$ (dd, $J=10.8$, $2.4 \mathrm{~Hz}, 1 \mathrm{H}), 6.75(\mathrm{td}, J=8.2,2.4 \mathrm{~Hz}, 1 \mathrm{H}), 7.10-7.13(\mathrm{~m}, 1 \mathrm{H}) ;{ }^{13} \mathrm{C} \mathrm{NMR}\left(\mathrm{CDCl}_{3}\right) \delta 27.36(\mathrm{~s})$, $31.21(\mathrm{p}), 45.68(\mathrm{~s}), 104.34\left(\mathrm{t}, J_{\mathrm{C}-\mathrm{F}}=26.5 \mathrm{~Hz}\right), 109.68\left(\mathrm{t}, J_{\mathrm{C}-\mathrm{F}}=21.5 \mathrm{~Hz}\right), 118.05\left(\mathrm{q}, J_{\mathrm{C}-\mathrm{F}}=4.2\right.$ $\mathrm{Hz}), 130.67\left(\mathrm{t}, J_{\mathrm{C}-\mathrm{F}}=9.1 \mathrm{~Hz}\right), 142.43\left(\mathrm{q}, J_{\mathrm{C}-\mathrm{F}}=9.9 \mathrm{~Hz}\right), 162.30\left(\mathrm{q}, J_{\mathrm{C}-\mathrm{F}}=245.6 \mathrm{~Hz}\right) ; \mathrm{MS}(\mathrm{EI})$ $\mathrm{M}^{+}$215; Anal. Calcd for $\mathrm{C}_{9} \mathrm{H}_{10} \mathrm{FNO}_{2} \mathrm{~S}: \mathrm{C}, 50.22 ; \mathrm{H}, 4.68 ; \mathrm{N}, 6.51$. Found: C, 50.03; H, 4.70; N, 6.50 .

$\mathrm{N}$-Methyl-7-fluoro-6-iodo-3,4-dihydro-2,1-benzothiazine 2,2-dioxide (3b). mp $115-117{ }^{\circ} \mathrm{C}$; IR (KBr) 3000, 2940, 1600, 1570, 1490, 1330, 1160, $1130 \mathrm{~cm}^{-1} ;{ }^{1} \mathrm{H}$ NMR $\left(\mathrm{CDCl}_{3}\right) \delta 3.27(\mathrm{~s}$, $3 \mathrm{H}), 3.34(\mathrm{t}, J=6.6 \mathrm{~Hz}, 2 \mathrm{H}), 3.41(\mathrm{t}, J=6.6 \mathrm{~Hz}, 2 \mathrm{H}), 6.66\left(\mathrm{~d}, J_{\mathrm{H}-\mathrm{F}}=9.6 \mathrm{~Hz}, 1 \mathrm{H}\right), 7.51\left(\mathrm{~d}, J_{\mathrm{H}-\mathrm{F}}\right.$ $=6.7 \mathrm{~Hz}, 1 \mathrm{H}) ;{ }^{13} \mathrm{C} \mathrm{NMR}\left(\mathrm{CDCl}_{3}\right) \delta 26.94(\mathrm{~s}), 31.01(\mathrm{p}), 45.41(\mathrm{~s}), 72.55\left(\mathrm{q}, J_{\mathrm{C}-\mathrm{F}}=26.3 \mathrm{~Hz}\right)$, $104.36\left(\mathrm{t}, J_{\mathrm{C}-\mathrm{F}}=27.9 \mathrm{~Hz}\right), 120.15\left(\mathrm{q}, J_{\mathrm{C}-\mathrm{F}}=3.3 \mathrm{~Hz}\right), 139.20\left(\mathrm{t}, J_{\mathrm{C}-\mathrm{F}}=6.6,2.5 \mathrm{~Hz}\right), 142.83(\mathrm{q}$, $\left.J_{\mathrm{C}-\mathrm{F}}=9.0 \mathrm{~Hz}\right), 161.17\left(\mathrm{q}, J_{\mathrm{C}-\mathrm{F}}=244.5 \mathrm{~Hz}\right)$; HRMS (EI) found $\mathrm{M}^{+}$340.9362, calcd for $\mathrm{C}_{9} \mathrm{H}_{9} \mathrm{FINO}_{2} \mathrm{~S} \mathrm{M} 340.9383$.

$\mathbf{N}$-Methyl-7-chloro-3,4-dihydro-2,1-benzothiazine 2,2-dioxide (2c). mp 93-94 ${ }^{\circ} \mathrm{C}$; IR ( $\left.\mathrm{KBr}\right)$ 3080, 2940, 1600, 1490, 1330, $1160 \mathrm{~cm}^{-1} ;{ }^{1} \mathrm{H}$ NMR $\left(\mathrm{CDCl}_{3}\right) \delta 3.29(\mathrm{~s}, 3 \mathrm{H}), 3.34(\mathrm{ddd}, J=8.2$, 5.6, $1.4 \mathrm{~Hz}, 2 \mathrm{H}), 3.43$ (t, $J=8.2,5.6,1.4 \mathrm{~Hz}, 2 \mathrm{H}), 6.93$ (d, $J=2.1 \mathrm{~Hz}, 1 \mathrm{H}), 7.02$ (dd, $J=8.2,2.1$ $\mathrm{Hz}, 1 \mathrm{H}), 7.09(\mathrm{~d}, J=8.2 \mathrm{~Hz}, 1 \mathrm{H}) ;{ }^{13} \mathrm{C} \mathrm{NMR}\left(\mathrm{CDCl}_{3}\right) \delta 27.53(\mathrm{~s}), 31.42(\mathrm{p}), 45.52$ (s), 117.10 (t), 120.89 (q), $122.96(\mathrm{t}), 130.45$ (t), 133.65 (q), 142.11 (q); MS (EI) $\mathrm{M}^{+}$231; Anal. Calcd for $\mathrm{C}_{9} \mathrm{H}_{10} \mathrm{ClNO}_{2} \mathrm{~S}: \mathrm{C}, 46.65 ; \mathrm{H}, 4.35$; N, 6.05. Found: C, 46.60; H, 4.29; N, 5.94.

$\mathrm{N}$-Methyl-7-chloro-6-iodo-3,4-dihydro-2,1-benzothiazine 2,2-dioxide (3c). mp $141-142{ }^{\circ} \mathrm{C}$; IR (KBr) 2980, 2940, 1580, 1550, 1460, 1330, 1160, $1130 \mathrm{~cm}^{-1} ;{ }^{1} \mathrm{H}$ NMR $\left(\mathrm{CDCl}_{3}\right) \delta 3.26(\mathrm{~s}$, $3 \mathrm{H}), 3.33(\mathrm{t}, J=6.8 \mathrm{~Hz}, 2 \mathrm{H}), 3.40(\mathrm{t}, J=6.8 \mathrm{~Hz}, 2 \mathrm{H}), 7.01(\mathrm{~s}, 1 \mathrm{H}), 7.61(\mathrm{~s}, 1 \mathrm{H})$; ${ }^{13} \mathrm{C}$ NMR $\left(\mathrm{CDCl}_{3}\right) \delta 27.01(\mathrm{~s}), 31.15(\mathrm{p}), 45.32(\mathrm{~s}), 89.59(\mathrm{q}), 117.33(\mathrm{t}), 122.74(\mathrm{q}), 137.90(\mathrm{q}), 140.13(\mathrm{t})$, 142.30 (q); MS (EI) $\mathrm{M}^{+}$357; Anal. Calcd for $\mathrm{C}_{9} \mathrm{H}_{9} \mathrm{ClINO}_{2} \mathrm{~S}: \mathrm{C}, 30.23$; H, 2.54; N, 3.92. Found: C, 30.30; H, 2.49; N, 3.83. 
$\mathrm{N}$-Methyl-6-iodo-7-methyl-3,4-dihydro-2,1-benzothiazine 2,2-dioxide (3d). mp $138-139{ }^{\circ} \mathrm{C}$; IR (KBr) 3000, 2940, 1600, 1550, 1330, $1160 \mathrm{~cm}^{-1} ;{ }^{1} \mathrm{H} \mathrm{NMR}_{\left(\mathrm{CDCl}_{3}\right) \delta} 2.41(\mathrm{~s}, 3 \mathrm{H}), 3.27(\mathrm{~s}$, $3 \mathrm{H}), 3.30(\mathrm{t}, J=6.8 \mathrm{~Hz}, 2 \mathrm{H}), 3.38(\mathrm{t}, J=6.8 \mathrm{~Hz}, 2 \mathrm{H}), 6.82(\mathrm{~s}, 1 \mathrm{H}), 7.57(\mathrm{~s}, 1 \mathrm{H}) ;{ }^{13} \mathrm{C} \mathrm{NMR}$ $\left(\mathrm{CDCl}_{3}\right) \delta 26.95(\mathrm{~s}), 27.89(\mathrm{p}), 31.86(\mathrm{p}), 45.31(\mathrm{~s}), 93.21(\mathrm{q}), 118.45(\mathrm{t}), 122.07$ (q), $139.06(\mathrm{t})$, 141.07 (q), 141.31 (q); MS (EI) $\mathrm{M}^{+}$337; Anal. Calcd for $\mathrm{C}_{10} \mathrm{H}_{12} \mathrm{INO}_{2} \mathrm{~S}: \mathrm{C}, 35.62 ; \mathrm{H}, 3.59 ; \mathrm{N}$, 4.15. Found: C, 35.67; H, 3.45; N, 3.99 .

$\mathbf{N}$-Methyl-3,4-dihydro-2,1-naphthothiazine 2,2-dioxide (2e). mp 190-191 ${ }^{\circ} \mathrm{C}$; IR (KBr) 3060, 2940, 1620, 1620, 1600, 1510, 1470, 1330, $1160 \mathrm{~cm}^{-1} ;{ }^{1} \mathrm{H} \mathrm{NMR}\left(\mathrm{CDCl}_{3}\right) \delta 3.43(\mathrm{~s}, 3 \mathrm{H}), 3.45(\mathrm{t}$, $J=7.1 \mathrm{~Hz}, 2 \mathrm{H}), 3.79(\mathrm{t}, J=7.1 \mathrm{~Hz}, 2 \mathrm{H}), 7.24(\mathrm{~d}, J=9.7 \mathrm{~Hz}, 1 \mathrm{H}), 7.48$ (ddd, $J=7.9,7.0,1.0 \mathrm{~Hz}$, $1 \mathrm{H}), 7.57$ (ddd, $J=8.5,7.0,1.4 \mathrm{~Hz}, 1 \mathrm{H}), 7.79$ (d, $J=9.7 \mathrm{~Hz}, 1 \mathrm{H}), 7.82$ (dd, $J=7.9,1.4 \mathrm{~Hz}, 1 \mathrm{H})$, $7.90(\mathrm{dd}, J=8.5,1.0 \mathrm{~Hz}, 1 \mathrm{H}) ;{ }^{13} \mathrm{C} \mathrm{NMR}\left(\mathrm{CDCl}_{3}\right) \delta 25.24(\mathrm{~s}), 35.77(\mathrm{p}), 43.30(\mathrm{~s}), 117.26(\mathrm{q})$, $119.72(\mathrm{t}), 122.30(\mathrm{t}), 125.27(\mathrm{t}), 127.34(\mathrm{t}), 128.62(\mathrm{t}), 128.99(\mathrm{t}), 130.21(\mathrm{q}), 131.71(\mathrm{q}), 139.07$ (q); MS (EI) $\mathrm{M}^{+}$247; Anal. Calcd for $\mathrm{C}_{13} \mathrm{H}_{13} \mathrm{NO}_{2} \mathrm{~S}$ : C, 63.13; H, 5.30; N, 5.66. Found: C, 63.10; $\mathrm{H}, 5.23 ; \mathrm{N}, 5.62$.

9-Benzenesulfonyl-1-methyl-3,4-dihydro-2,1-indolethiazine 2,2-dioxide (2f). $\mathrm{mp} \quad 208{ }^{\circ} \mathrm{C}$ (decomp.); IR (KBr) 3100, 3080, 2930, 1630, 1460, 1360, $1180 \mathrm{~cm}^{-1} ;{ }^{1} \mathrm{H} \mathrm{NMR}\left(\mathrm{CDCl}_{3}\right) \delta 3.08$ $(\mathrm{t}, J=6.7 \mathrm{~Hz}, 2 \mathrm{H}), 3.19(\mathrm{t}, J=6.7,2 \mathrm{H}), 3.43(\mathrm{~s}, 3 \mathrm{H}), 7.22-7.27(\mathrm{~m}, 2 \mathrm{H}), 7.30-7.35(\mathrm{~m}, 3 \mathrm{H})$, $7.44(\mathrm{tt}, J=7.5,1.4 \mathrm{~Hz}, 1 \mathrm{H}), 7.80-7.82(\mathrm{~m}, 2 \mathrm{H}), 8.19(\mathrm{~d}, J=8.5 \mathrm{~Hz}, 1 \mathrm{H}) ;{ }^{13} \mathrm{C} \mathrm{NMR}\left(\mathrm{CDCl}_{3}\right) \delta$ $19.48(\mathrm{~s}), 38.97(\mathrm{~s}), 43.02(\mathrm{p}), 107.24(\mathrm{q}), 116.19(\mathrm{t}), 118.05(\mathrm{t}), 124.74(\mathrm{t}), 125.26(\mathrm{t}), 127.29(\mathrm{t})$, $128.48(\mathrm{q}), 128.81(\mathrm{t}), 134.04(\mathrm{t}), 134.51$ (q), 136.26 (q), 136.87 (q); HRMS (EI) found $\mathrm{M}^{+}$ 376.0553, calcd for $\mathrm{C}_{17} \mathrm{H}_{16} \mathrm{~N}_{2} \mathrm{O}_{4} \mathrm{~S}_{2} \mathrm{M} 376.0552$.

3-Iodo-9-benzenesulfonyl-1-methyl-2,1-indolethiazine 2,2-dioxide (4). mp $195{ }^{\circ} \mathrm{C}$ (decomp.); IR (KBr) 3070, 2960, 2920, 1730, 1590, 1580, 1370, 1350, 1180, $1170 \mathrm{~cm}^{-1} ;{ }^{1} \mathrm{H} \mathrm{NMR}\left(\mathrm{CDCl}_{3}\right)$ $\delta 3.54(\mathrm{~s}, 3 \mathrm{H}), 7.26-7.34(\mathrm{~m}, 3 \mathrm{H}), 7.37-7.41(\mathrm{~m}, 2 \mathrm{H}), 7.46(\mathrm{tt}, J=7.5,1.2 \mathrm{~Hz}, 1 \mathrm{H}), 7.76(\mathrm{~s}, 1 \mathrm{H})$, $7.81(\mathrm{dd}, J=8.7,1.1 \mathrm{~Hz}, 2 \mathrm{H}), 8.18(\mathrm{~d}, J=8.5 \mathrm{~Hz}, 1 \mathrm{H}) ;{ }^{13} \mathrm{C} \mathrm{NMR}\left(\mathrm{CDCl}_{3}\right) \delta 41.02(\mathrm{p}), 80.64$ $(\mathrm{q}), 111.24(\mathrm{q}), 116.43(\mathrm{t}), 118.24(\mathrm{t}), 125.38(\mathrm{q}), 125.59(\mathrm{t}), 126.03(\mathrm{t}), 127.49(\mathrm{t}), 129.05(\mathrm{t})$, $134.58(\mathrm{t}), 134.74(\mathrm{t}), 135.68(\mathrm{q}), 136.42(\mathrm{t}), 140.22(\mathrm{q})$; HRMS (EI) found $\mathrm{M}^{+}$499.9352, calcd for $\mathrm{C}_{17} \mathrm{H}_{13} \mathrm{IN}_{2} \mathrm{O}_{4} \mathrm{~S}_{2} \mathrm{M} 499.9362$.

Typical procedure for the ionic cyclization of $N$-methoxy-2-phenylethanesulfonamide with PSHTIB

$\mathrm{N}$-Methoxy-2-phenylethanesulfonamide $(0.5 \mathrm{mmol})$ was added to a solution of PSHTIB $(0.75$ $\mathrm{mmol})$ in acetonitrile $(5 \mathrm{~mL})$. The mixture was stirred at room temperature for $4 \mathrm{~h}$ under an argon atmosphere. After the reaction, diethyl ether $(10 \mathrm{~mL})$ was added to the reaction mixture and the mixture was filtered to remove poly(4-iodostyrene). The filtrate was poured into water and extracted with diethyl ether thrice. The combined organic layer was dried over sodium sulfate. After filtration, the solvent was removed and the residue was chromatographed on silica gel (eluent: hexane / ethyl acetate $=2 / 1$ ).

$\mathbf{N}$-Methoxy-3,4-dihydro-2,1-benzothiazine 2,2-dioxide (6a). mp 104-106 ${ }^{\circ} \mathrm{C}$; IR (KBr) 3000, 2950, 2815, 1580, 1480, 1360, $1170 \mathrm{~cm}^{-1} ;{ }^{1} \mathrm{H} \mathrm{NMR}\left(\mathrm{CDCl}_{3}\right) \delta 3.42(\mathrm{t}, J=6.4 \mathrm{~Hz}, 2 \mathrm{H}), 3.50(\mathrm{td}$, 
$J=6.4,1.5 \mathrm{~Hz}, 2 \mathrm{H}), 4.08(\mathrm{~s}, 3 \mathrm{H}), 7.20-7.23(\mathrm{~m}, 2 \mathrm{H}), 7.31-7.34(\mathrm{~m}, 1 \mathrm{H}), 7.36-7.40(\mathrm{~m}, 2 \mathrm{H}) ;{ }^{13} \mathrm{C}$ $\operatorname{NMR}\left(\mathrm{CDCl}_{3}\right) \delta 27.86(\mathrm{~s}), 40.20(\mathrm{~s}), 65.57(\mathrm{p}), 126.72(\mathrm{q}), 127.88(\mathrm{t}), 128.03(\mathrm{t}), 128.90(\mathrm{t})$, 129.41 (t), 141.88 (q); MS (EI) M ${ }^{+} 213$; Anal. Calcd for $\mathrm{C}_{9} \mathrm{H}_{11} \mathrm{NO}_{3} \mathrm{~S}: \mathrm{C}, 50.69 ; \mathrm{H}, 5.20 ; \mathrm{N}, 6.57$. Found: C, 50.76; H, 5.32; N, 6.58 .

$\mathrm{N}$-Methoxy-7-fluoro-3,4-dihydro-2,1-benzothiazine 2,2-dioxide (6b). mp $76-78{ }^{\circ} \mathrm{C}$; IR ( $\mathrm{KBr}$ ) 3000, 2950, 2820, 1600, 1490, 1360, $1170 \mathrm{~cm}^{-1} ;{ }^{1} \mathrm{H} \mathrm{NMR}\left(\mathrm{CDCl}_{3}\right) \delta 3.37(\mathrm{t}, J=6.4 \mathrm{~Hz}, 2 \mathrm{H})$, $3.47(\mathrm{td}, J=6.4,1.3 \mathrm{~Hz}, 2 \mathrm{H}), 4.07(\mathrm{~s}, 3 \mathrm{H}), 7.02(\mathrm{td}, J=8.2,2.7 \mathrm{~Hz}, 1 \mathrm{H}), 7.08(\mathrm{dd}, J=8.8,2.7$ $\mathrm{Hz}, 1 \mathrm{H}), 7.17(\mathrm{dd}, J=8.6,6.0 \mathrm{~Hz}, 1 \mathrm{H}) ;{ }^{13} \mathrm{C} \mathrm{NMR}\left(\mathrm{CDCl}_{3}\right) \delta 27.22(\mathrm{~s}), 40.48(\mathrm{~s}), 65.83(\mathrm{p})$, $113.52\left(\mathrm{t}, J_{\mathrm{C}-\mathrm{F}}=23.8 \mathrm{~Hz}\right), 115.96\left(\mathrm{t}, J_{\mathrm{C}-\mathrm{F}}=22.1 \mathrm{~Hz}\right), 122.14\left(\mathrm{q}, J_{\mathrm{C}-\mathrm{F}}=4.2 \mathrm{~Hz}\right), 130.72\left(\mathrm{t}, J_{\mathrm{C}-\mathrm{F}}\right.$ $=8.2 \mathrm{~Hz}), 142.84\left(\mathrm{q}, J_{\mathrm{C}-\mathrm{F}}=9.8 \mathrm{~Hz}\right), 161.64\left(\mathrm{q}, J_{\mathrm{C}-\mathrm{F}}=247.5 \mathrm{~Hz}\right) ; \mathrm{MS}(\mathrm{EI}) \mathrm{M}^{+} 231$; Anal. Calcd for $\mathrm{C}_{9} \mathrm{H}_{10} \mathrm{FNO}_{3} \mathrm{~S}$ : C, 46.75; H, 4.36; N, 6.06. Found: C, 46.94; H, 4.41; N, 5.89.

$\mathrm{N}$-Methoxy-7-chloro-3,4-dihydro-2,1-benzothiazine 2,2-dioxide (6c). mp $123-125{ }^{\circ} \mathrm{C}$; IR (KBr) 3000, 2950, 2815, 1600, 1480, 1360, $1160 \mathrm{~cm}^{-1} ;{ }^{1} \mathrm{H} \mathrm{NMR}\left(\mathrm{CDCl}_{3}\right) \delta 3.37$ (t, $J=6.7 \mathrm{~Hz}$, 2H), 3.48 (t, $J=6.5 \mathrm{~Hz}, 2 \mathrm{H}), 4.08$ (s, 3H), 7.14 (d, $J=8.2 \mathrm{~Hz}, 1 \mathrm{H}), 7.27$ (dd, $J=8.5,2.0 \mathrm{~Hz}$, $1 \mathrm{H}), 7.36(\mathrm{~d}, J=2.1 \mathrm{~Hz}, 1 \mathrm{H}) ;{ }^{13} \mathrm{C} \mathrm{NMR}\left(\mathrm{CDCl}_{3}\right) \delta 27.55(\mathrm{~s}), 40.42(\mathrm{~s}), 65.95(\mathrm{p}), 125.08(\mathrm{q})$, $127.14(\mathrm{t}), 128.92$ (t), 130.60 (t), 133.53 (q), 142.90 (q); MS (EI) $\mathrm{M}^{+}$247; Anal. Calcd for $\mathrm{C}_{9} \mathrm{H}_{10} \mathrm{ClNO}_{3} \mathrm{~S}: \mathrm{C}, 43.64 ; \mathrm{H}, 4.07 ; \mathrm{N}, 5.65$. Found: C, 43.39; H, 4.08; N, 5.52.

$\mathrm{N}$-Methoxy-7-methyl-3,4-dihydro-2,1-benzothiazine 2,2-dioxide (6d). mp $119-121{ }^{\circ} \mathrm{C}$; IR (KBr) 3000, 2950, 2815, 1620, 1500, 1360, $1170 \mathrm{~cm}^{-1} ;{ }^{1} \mathrm{H} \mathrm{NMR}\left(\mathrm{CDCl}_{3}\right) \delta 2.36(\mathrm{~s}, 3 \mathrm{H}), 3.36(\mathrm{t}$, $J=6.6 \mathrm{~Hz}, 2 \mathrm{H}), 3.47$ (t, $J=6.6 \mathrm{~Hz}, 2 \mathrm{H}), 4.07$ (s, 3H), 7.08 (d, $J=7.9 \mathrm{~Hz}, 1 \mathrm{H}), 7.12$ (dd, $J=7.6$, $1.6 \mathrm{~Hz}, 1 \mathrm{H}), 7.18(\mathrm{~s}, 1 \mathrm{H}) ;{ }^{13} \mathrm{C} \mathrm{NMR}\left(\mathrm{CDCl}_{3}\right) \delta 20.99(\mathrm{p}), 27.56(\mathrm{~s}), 40.20(\mathrm{~s}), 65.68(\mathrm{p}), 123.61$ (q), 128.27 (t), 129.32 (t), 130.07 (t), 138.22 (q), 141.63 (q); MS (EI) $\mathrm{M}^{+}$227; Anal. Calcd for $\mathrm{C}_{10} \mathrm{H}_{13} \mathrm{NO}_{3} \mathrm{~S}$ : C, 52.85; H, 5.77; N, 6.16. Found: C, 52.80; H, 5.69; N, 6.14.

$N$-Methoxy-2,2-dioxo-2 $\lambda^{6}$-thia-1-azaspiro[4.5]deca-6,9-dien-8-one (7). mp $133-135^{\circ} \mathrm{C}$; IR (KBr) 2980, 2950, 2830, 1680, 1610, 1400, 1330, $1160 \mathrm{~cm}^{-1} ;{ }^{1} \mathrm{H}$ NMR $\left(\mathrm{CDCl}_{3}\right) \delta 2.44(\mathrm{t}, J=8.0$ $\mathrm{Hz}, 2 \mathrm{H}), 3.44$ (t, $J=7.9 \mathrm{~Hz}, 2 \mathrm{H}), 3.81(\mathrm{~s}, 3 \mathrm{H}), 6.39$ (d, $J=10.1 \mathrm{~Hz}, 2 \mathrm{H}), 7.05$ (d, $J=10.4 \mathrm{~Hz}$, $2 \mathrm{H}) ;{ }^{13} \mathrm{C} \mathrm{NMR}\left(\mathrm{CDCl}_{3}\right) \delta 27.19(\mathrm{~s}), 43.15(\mathrm{~s}), 61.79(\mathrm{q}), 66.62(\mathrm{p}), 131.12(\mathrm{t}), 145.74(\mathrm{t}), 184.14$ (q); MS (EI) $\mathrm{M}^{+} 229$; Anal. Calcd for $\mathrm{C}_{9} \mathrm{H}_{11} \mathrm{NO}_{4} \mathrm{~S}: \mathrm{C}, 47.15 ; \mathrm{H}, 4.84 ; \mathrm{N}, 6.11$. Found: $\mathrm{C}, 47.16$; $\mathrm{H}, 4.68 ; \mathrm{N}, 6.07$.

\section{Procedure for the ionic cyclization of $N$-methoxy-3-phenylpropionamide with PSHTIB}

A mixture of $N$-Methoxy-3-phenylpropionamide $(0.5 \mathrm{mmol})$ in acetonitrile $(40 \mathrm{~mL})$ was added dropwise to a solution of PSHTIB $(0.75 \mathrm{mmol})$ in acetonitrile $(10 \mathrm{~mL})$. The mixture was stirred at room temperature for $5 \mathrm{~h}$ under an argon atmosphere. After the reaction, the reaction mixture was filtered to remove the polymer species. The filtrate was evaporated and the residue was extracted with water and diethyl ether thrice. The combined organic layer was dried over sodium sulfate. After filtration, the solvent was removed and the residue was chromatographed on silica gel (eluent: hexane / ethyl acetate $=2 / 1$ ).

$\mathrm{N}$-Methoxy-3,4-dihydro-1H-quinolin-2-one (9). oil; IR (neat) 2940, 2840, 1690 (br), 1610, 1490, 1460, 1360, 1270, 1190, 960, $760 \mathrm{~cm}^{-1} ;{ }^{1} \mathrm{H}$ NMR $\left(\mathrm{CDCl}_{3}\right) \delta 2.68-2.74(\mathrm{~m}, 2 \mathrm{H}), 2.92(\mathrm{t}, J$ 
$=6.9 \mathrm{~Hz}, 2 \mathrm{H}), 3.92(\mathrm{~s}, 3 \mathrm{H}), 7.04(\mathrm{td}, J=7.3,1.3 \mathrm{~Hz}, 1 \mathrm{H}), 7.17(\mathrm{dd}, J=7.3,0.9 \mathrm{~Hz}, 1 \mathrm{H}), 7.23$ (dd, $J=8.1,1.3 \mathrm{~Hz}, 1 \mathrm{H}), 7.26-7.32(\mathrm{~m}, 1 \mathrm{H})$. MS (EI) $\mathrm{M}^{+} 177$.

\section{References}

1. (a) Varvoglis, A. Synthesis 1984, 709. (b) Stang, P. J. Angew. Chem., Int. Ed. 1992, 31, 274. (c) Stang, P. J.; Zhankin, V. V. Chem. Rev. 1996, 96, 1123. (d) Muraki, T.; Togo, H.; Yokoyama, M. Rev. Heteroatom Chem. 1997, 17, 213. (e) Zhankin, V. V. Rev. Heteroatom Chem. 1997, 17, 133. (f) Varvoglis, A. Tetrahedron 1997, 53, 1179.

2. Yamada, Y.; Okawara, M. Die Makromol. Chem. 1972, 152, 153.

3. (a) Togo, H.; Nogami, G.; Yokoyama, M. Synlett 1998, 534. (b) Ley, S. V.; Thomas, A. W.; Finch, H. J. Chem. Soc., Perkin Trans. 1 1999, 669. (c) Boehlow, T. R.; Harburn, J. J.;Spilling, C. D. J. Org. Chem. 2001, 66, 3111. (d) Ficht, S.; Mülbaier, M.; Giannis, A. Tetrahedron 2001, 57, 4863. (e) Tohma, H.; Morioka, H.; Takizawa, S.; Arisawa, M.; Kita, Y. Tetrahedeon 2001, 57, 345.

4. (a) Zupan, M.; Pollak, A. J. Chem. Soc., Chem. Comm. 1975, 715. (b) Šket, B.; Zupan, M.; Zupet, P. Tetrahedron 1984, 40, 1603. (c) Moss, R. A.; Chung, Y.-C. J. Org. Chem. 1990, 55, 2064. (d) Huang, X.; Zhu, Q. Tetrahedron Letters 2001, 42, 6373. (e) Mülbaier, M.; Giannis, A. Angew. Chem., Int. Ed. 2001, 40, 4393. (f) Abe, S.; Sakuratani, K.; Togo, H. J. Org. Chem. 2001, 66, 6174. (g) Togo, H.; Sakuratani, K. Synlett 2002, 1966.

5. (a) Togo, H.; Muraki, T.; Hoshina, Y.; Yamaguchi, K.; Yokoyama, M. J. Chem. Soc., Perkin Trans. 1 1997, 787. (b) Togo, H.; Hoshina, Y.; Muraki, T.; Nakayama, H.; Yokoyama, M. J. Org. Chem. 1998, 63, 5193. (c) Katohgi, M.; Togo, H.; Yamaguchi, K.; Yokoyama, M. Tetrahedron 1999, 55, 14885. (d) Muraki, T.; Togo, H.; Yokoyama, M. J. Org. Chem. 1999, 64, 2883. (e) Togo, H.; Harada, Y.; Yokoyama, M. J. Org. Chem. 2000, 65, 926.

6. (a) Kaiser, E. M.; Knutsonm P. L. A. J. Org. Chem. 1975, 40, 1342. (b) Cecchetti, V.; Fravolini, A.; Schiaffella, F. J. Heterocycl. Chem. 1982, 19, 1045. (c) Chem. Abstr. 1985, 102, 605 (78901p, JP 59164786). (d) Chem. Abstr. 1992, 117, 748 (131207e, WO 9205164). (e) Chem. Abstr. 1990, 112, 585 (35887e, JP 0161470). (f) Harmata, M.; Kahraman, M. J. Org. Chem. 1998, 63, 6845.

7. (a) Loev, B.; Kormendy, M. F. J. Org. Chem. 1965, 30, 3163. (b) Loev, B.; Kormendy, M. F.; Snader, K. M. J. Org. Chem. 1966, 31, 3531. (c) Abramovitch, R. A.; Holcomb, W. D. J. Am. Chem. Soc. 1975, 97, 676. (d) Blondet, D.; Pascal, J.-C. Tetrahedron Lett. 1994, 35, 2911.

8. Kikugawa, Y.; Kawase, M. Chem. Lett. 1990, 581.

9. Rudolph, A. A.; William, D. H. J. Am. Chem. Soc. 1981, 103, 1525. 